\title{
MeerKAT and its potential for Cosmic MASER Research
}

\author{
Roy Booth ${ }^{1,2}$, Sharmila Goedhart ${ }^{1}$ and Justin Jonas ${ }^{2}$ \\ ${ }^{1}$ SKA South Africa, The Park, Park Road, Pinelands, 7005, South Africa, \\ ${ }^{2}$ Rhodes University, P.O Box 94, Grahamstown 6140, South Africa \\ email: rbooth@ska.ac.za
}

\begin{abstract}
The MeerKAT radio telescope array is the South African precursor instrument for the proposed Square Kilometer Array's mid-band frequency range. It will be the most sensitive centimetre-wavelength telescope in the southern hemisphere until the SKA is built. It will cover a broad range of astronomical science from the evolution of galaxies to tests of Einstein's theory of General Relativity, using Pulsars. The chosen frequency bands will enable sensitive southern Galactic maser surveys in the main lines of hydroxyl, $12 \mathrm{GHz}$ methanol and perhaps14.5 $\mathrm{GHz}$ formaldehyde lines as well as searches for redshifted water masers from red-shifts greater than about 0.5. Proposals for Large Surveys using MeerKAT were solicited in late 2009 and resulted in some 20 proposals from teams comprising 500 scientists, world-wide. The successful proposals relevant to maser research will be discussed below.
\end{abstract}

Keywords. radio telescope design, arrays, senstivity, surveys, masers

\section{Introducion}

MeerKAT is the South African SKA precursor telescope array. Its development began with a prototype $15 \mathrm{~m}$ paraboloid antenna, XDM, of innovative, light-weight design, the dish being cast in a single piece from composite (fibre-glass) material on a mould built on site at the Hartebeesthoek Radio Astronomy Observatory. The mould had been flame sprayed with a thin layer of aluminium before depositing the composite. The antenna performed well, having a surface rms of better than $2 \mathrm{~mm}$, and set the scene for the first 7-element test array (KAT-7) for which all the (12 m diameter) dishes were cast at a base near the remote site in the Northern Cape region known as the Karoo, removing the need for long distance transportation. KAT-7 operates in the frequency range $1.2-1.95 \mathrm{GHz}$ and is currently undergoing science commissioning and producing useful data. The receivers are cooled with Stirling-cycle coolers to about $70 \mathrm{~K}$. Data signal processing is conducted using another innovative development, to which the KAT engineers have made a serious contribution. This is the ROACH (reconfigurable open architecture computing hardware) board which has become the primary building block for digital signal processing in several next-generation radio telescopes. As this conference draws to a close, the first narrow band correlator modes, suitable for $(\mathrm{OH})$ MASER research are being tested.

\section{The MeerKAT Concept}

The experience gained from XDM and KAT-7 has been factored into the MeerKAT array design. The goal is to build the world's most sensitive centimetre wavelength radio telescope in the southern hemisphere, until the SKA becomes a reality and, after a 
design review in 2010, an ambitious concept of 64 x 13.5 m offset Gregorian antennas was conceived. The array will perform high dynamic range, high-fidelity imaging over nearly an order of magnitude in resolution, with a resolving power of 6 arcseconds at $1420 \mathrm{MHz}$. It will be optimised for deep, high fidelity imaging of extended low brightness emission, the detection of micro-Jansky radio sources, the measurement of polarization and the detection and monitoring of transient radio sources. Ultimately, the array will operate over a frequency range of $580 \mathrm{MHz}$ to $14.5 \mathrm{GHz}$ but in the first phase (2016) a band of $900-1670 \mathrm{MHz}$ is planned. The other receiver bands will be phased as shown below.

The high sensitivity criterion implies a large effective collecting area and a low system temperature, or maximisation of $\mathrm{A}_{e} / \mathrm{T}_{\text {sys }}$ - the goal is circa $300 \mathrm{~m}^{2}$ per kelvin at L-band with cooled $(20 \mathrm{k})$ receivers. This, together with a high dynamic range, are the hallmarks of a powerful interferometer array. To achieve this goal the individual parameters should not be compromised. So, for example, rather than go for the widest band feeds, which compromise $\mathrm{T}_{\text {sys }}$ at the band edges, it is preferable to use somewhat narrower bandwidths. To maximise $\mathrm{A}_{e}$ we have chosen an offset Gregorian antenna design, giving an unblocked aperture and high aperture illumination without introducing high, or polarised sidelobes.

A further advantage of the offset antenna is that the arm carrying the secondary reflector can be designed to carry the individual cooled receivers thus alleviating the need to change receivers when switching between observing bands. A receiver indexer at the secondary focal position will have capacity for up to 4 receivers.

Finally, in another bold move, the MeerKAT digital engineers are working to eliminate the intermediate frequency receiver stage by direct digitisation of the entire RF band, after initial amplification in a low noise receiver. This is currently feasible at L-band but represents a significant challenge for the highest frequency $(8-14.5 \mathrm{GHz})$ band. However they are confident that it wil be possible by 2016-17.

\section{The Array configuration and receiver Phasing}

As an array telescope, MeerKAT can and will be built in phases and early science will be possible before the full 64 antennae are erected; the receivers covering the different frequency bands will be deployed in a serial manner, as summarised below.

\subsection{Phase 1}

The array configuration will have a maximum baseline of $8 \mathrm{~km}$ and $70 \%$ of the collecting area will be within a $1 \mathrm{~km}$ diameter core to provide high brightness temperature sensitivity and reduce computational load for transient source data processing. The Phase 1 receiver will cover the frequency range 0.9-1.67 GHz and facilitate early continuum and red-shifted $\mathrm{H} 1$ and $\mathrm{OH}$ (maser) observations. Direct sampling at RF will be implemented, with all signal processing being performed in the digital domain using hardware and algoritms developed as part of the CASPER collaboration.

\subsection{Future phases}

The capabilities of MeerKAT will be expanded, as funding allows, and the frequency coverage of the instrument will be extended with the installation of up to three additional receivers:

(a) 580 - $1000 \mathrm{MHz}$ : This receiver will define the redshift range of the deep HI/OH surveys and will be used to observe steep-spectrum continuum sources. 
(b) 8 - $14.5 \mathrm{GHz}$ (goal: 4-16 GHz): This wide bandwidth receiver will allow the detection of highly redshifted $\mathrm{CO}$ emission, red-shifted water masers from $\mathrm{z}=0.5$, as well as Galactic maser species due to methanol, formaldehyde and excited $\mathrm{OH}$, and will facilitate the detection and monitoring of pulsars in the Galactic centre, and the mapping of the emission from a variety of molecules.

(c) 1.5 - $3 \mathrm{GHz}$ : The primary use of this receiver would be the precision timing of pulsars for the detection of gravitational waves.

Finally, it is imperative that funding is found for several additional antennas to extend the maximum baselines of the array out to some $20 \mathrm{~km}$ or more. Such an extension will improve the resolution of the array so that the deep continuum survey will be able to probe to lower flux densities without being affected by source confusion and newly detected sources may be located to within about 1 arcsecond to allow follow-up observations at visible and other wavelengths. Further expansion of the array to increase the collecting area (and perhapsresolution) will depend on the decisions related to the implementation of the SKA.

\section{MeerKAT Science: The Large Survey Projects}

MeerKAT is one of a number of new or re-scoped radio astronomy arrays under construction/test at the moment. These include the ALMA in Chile, EVLA in the USA, E-MERLIN in the UK, LOFAR and APERTIF in The Netherlands, and ASKAP, in Australia. There is considerable synergy among these projects and at least four have chosen to allocate a large fraction of their observing time in the form of Large Survey Projects, which have generated considerable interest among members of the astronomical community. In the case of MeerKAT, we decided to allocate $70 \%$ of the observing time in this way, with the remaining $30 \%$ being reserved for PI projects $(\approx 20 \%)$ and Directors discretionary/urgent observations $(\approx 10 \%)$.

The MeerKAT call for Large Survey Project proposals (Booth et al. 2009) returned 20 excellent and timely project ideas from a total of 700 astronomers world-wide (some 500 individuals, including 68 South African astronomers). The total time requested amounted to about 10 years of full-time observing and so we set up a Time Allocation Committee (TAC) consisting of ten international experts to adjudicate and rank the proposals. Ten of the proposals were rated as outstanding and allocated observing time (amounting, in total, to 5 years). The most highly rated proposals were Pulsar timing observations related to gravitational radiation (Observations in the highest frequency band where interstellar scattering is unlikely to be a serious problem) (PI Matthew Bailes) and a deep HI survey to detect HI (and $\mathrm{OH}$ ) at the highest possible red-shift: $z=1.4$ at 580 $\mathrm{MHz}$ (Acronym LADUMA). (see below). The other highly rated proposals were related to Pulsars and Transient Radio Sources, Deep continuum observations, spectroscopy and VLBI. Of these we will discuss only those of relevance to MASER research.

\section{1. $H I$ (and $O H)$ Galaxy Surveys}

\subsubsection{LADUMA}

- PIs: Blythe (UCT), Baker (Rutgers) and Holweda (ESA)

This group will study HI and its properties at red-shifts out to the maximum MeerKAT can reach $(z=1.4)$ towards the Chandra Deep Field (CDFS). They will need to use the technique of "stacking" to detect the weak HI emission and this requires that they know the red-shift from surveys at other wavelengths (e.g. optical and IR). HDF-south is a well studied region at many wavelengths and probably one of the best regions for this 
kind of work. The detection of $\mathrm{OH}$ molecules and masers will be incidental but certainly a bi-product (sometimes a confusing bi-product) of this survey

\subsubsection{Galaxy Clustering}

- PI: Paolo Serra (ASTRON)

Fornax is the second most massive cluster within $20 \mathrm{Mpc}$ from the Sun and the largest cluster in the southern hemisphere. Its low X-ray luminosity makes it representative of the environment where most galaxies live. Furthermore, Fornax's ongoing growth makes it an excellent laboratory for the study of structure formation. Ideally located for MeerKAT observation, it provides an excellent opportunity to study the assembly of clusters, the physics of the accretion and stripping of gas in galaxies falling in the cluster, and to probe for the first time the connection between these processes and the neutral medium in the cosmic web.

\subsection{3. $M H O N G O O S E$}

\section{- PI: de Blok (ASTRON)}

This is a essentially a continuation of an earlier study of $\mathrm{HI}$ (and $\mathrm{OH}$ ) in nearby galaxies of different types, with the VLA - THINGS. Among thelarge array of interesting studies in this proposal is the connection between star formation and HI dynamics and accretion. Of particular interest here is the study of the outer discs of galaxies and the Cosmic web, and galaxy halos and dark matter. The data will be compared with CO observations from ALMA and other Millimetre arrays, as well as Spitzer (IR) data, building up a picture of the relative distribution and importance of atomic and molecular hydrogen in the evolution of galaxies.

\subsubsection{Absorption Line Survey}

- PIs: Gupta (ATNF) and Srianand (IUCAA)

Since it is easier to detect small quantities of cool gas in absorption against a continuum radio source, than in emission, this is another way to detect HI, even the hydroxyl radical $(\mathrm{OH})$ at high red-shift. Given sufficient frequency resolution, it is also an excellent way to detect the line splitting caused by the Zeeman effect and to measure magnetic fields. A further interesting study that will be conducted as part of this project is the constancy of Fundamental Constants as a function of $\mathrm{z}$ using $\mathrm{OH}$ lines and their different dependencies onthe fine structure constant and the electron/proton mass ratio .

\subsection{Spectroscopic observations with the high frequency receiver (8-14.5 GHz)}

\subsubsection{MESMER}

- PI: Heywood (Oxford)

Carbon monoxide $(\mathrm{CO})$ is the next most abundant molecule after molecular hydrogen $\left(\mathrm{H}_{2}\right) . \mathrm{H}_{2}$ is difficult to observe in the ground state because it has no dipole moment and therefore has no rotational spectral lines. $\mathrm{H}_{2}$ is usually detected only in the IR or UV, when highly excited.

CO has been detected in a galaxy with a red shift of 6.4 (SDSS J1148+52), and is commonly detected at $z=4$, or less, showing that molecular hydrogen, perhaps unexpectedly, has a relatively high abundance in the early Universe. Theoretical studies have shown that molecular hydrogen may be more abundant in the early universe where galaxies may be more tightly wound, and the pressure and density may be relatively high. It is considered important to follow up on these, perhaps unexpected, discoveries and the highest frequency of MeerKAT was chosen for precisely this reason. The upper frequency of MeerKAT, $14.5 \mathrm{GHz}$ is equivalent to the CO line red-shifted to $z \approx 7$. 


\subsection{2. $\operatorname{Meer} G A L$}

- PIs: Thompson (Hertfordshire) and Goedhart (SKA SA Project)

MeerKAT and its high frequency receiver will be unique in the southern hemisphere for studies of the southern Galaxy and the Magellanic Clouds. The MeerGAL study will measure the properties of the Galaxy, its stellar formation and evolution through continuum and line observations of HII regions, recombination lines and simple molecules. The recombination line work will give improved data on the Galactic rotation curve in the southern Galaxy. Interstellar methanol masers at $12 \mathrm{GHz}$ are giving us important data on Galactic astrometry (e.g. the distance to the Galactic centre) and the discovery and utilisation of new southern hemisphere masers will be very important in this, and other respects. Finally, the 8-14.5 GHz spectral region has not been studied in detail for new molecules or masers, and especially astro-biologically important molecules.

\section{The way forward}

The MeerKAT organization is anxious to ensure that the Survey Teams will work with the project to ensure a coherent approach to issues like Project Development, Special Software, Data Format (we propose VO compatibility), Team Organization and Dynamics (working groups), Publication/Data Release Policy and Outreach.

We will hold regular (annual in the first instance) meetings of PIs (the first was in February 2011) and request that the Project teams conduct annual meetings with our scientific/technical liaison personnel to report progress, problems and possible changes in scientific priorities as other projects progress. We are also encouraging projects to set up a coherent organization within their projects.

The MeerKAT project will adapt its HR policy towards hiring special postdocs and $\mathrm{PhD}$ positions in fields related to the accepted Large Survey Programmes, and has requested that a reciprocal policy is adopted by the team leaders.

Finally, in due course we will issue calls for proposals for the $20 \%$ of time to be devoted to PI proposals and MASERS will indoubtedly be the theme of many of these.

\section{References}

Booth, R. S., de Blok, W. J. G., Jonas, J. L., \& Fanaroff, B. 2009, arXiv 0910.2935B 